\title{
QUADRATIC MODULES BIFIBRED OVER NIL(2)-MODULES
}

\author{
Hasan Atik and Erdal Ulualan
}

\begin{abstract}
In this work, we show that the forgetful functor from the category of Baues's quadratic modules to that of nil(2)-modules is a bifibration.
\end{abstract}

\section{Introduction}

Quadratic modules were introduced by Baues, 4, as algebraic models for homotopy connected 3-types. They are related to simplicial groups, crossed squares [15, 16] and 2-crossed modules 12 as explained in [3, 4]. The freeness conditions for quadratic modules of groups in terms of free simplicial groups were also described in [2].

Brown and Sivera [8] have shown that the forgetful functor $\Phi_{1}:$ XMod $\rightarrow$ Gpd from the category of crossed modules of groupoids to the category of groupoids, which sends a crossed module $M \rightarrow P$ to its base groupoid $P$, is a fibration and also a cofibration of categories. This result in the case of crossed modules of groups was appeared in [5] and pursued in [9, 10]. This allows detailed computations of non-Abelian information on second relative groups. Analogous constructions in the crossed modules category in Lie algebras and commutative algebras are given in [11] and [18, respectively. For further accounts of fibred and cofibred categories and introduction to their literature, see [7, 13, 20] and the references there.

In this paper, to shed some light on the quadratic module structure, we extend the result of Brown and Sivera to the forgetful functor $\Phi:$ Quad $\rightarrow \operatorname{Nil}(2)$ from the category of quadratic modules to that of nil(2)-modules. Thus, the main aim of this paper is to show that quadratic modules are fibred and cofibred over nil(2)-modules. This gives the notions of pullback and induced quadratic module by a morphism of nil(2)-modules. Clearly, this result and general methods given for quadratic modules are 2-dimensional analogues of the description of the pullback and induced crossed modules given in [5, 6, 7, 8, 11, 18, 


\section{Basic Definitions}

\subsection{Fibration and Cofibration of Categories}

The definitions and properties related to fibrations and cofibrations of categories, some of which are classical, are recalled from [8].

Definition 1.1 Let $\Phi: \mathrm{X} \rightarrow \mathrm{B}$ be a functor. A morphism $\varphi: Y \rightarrow X$ in $\mathrm{X}$ over $u:=\Phi(\varphi)$ is called cartesian if and only if for all $v: K \rightarrow J$ in $\mathrm{B}$ and $\theta: Z \rightarrow X$ with $\Phi(\theta)=$ uv there is a unique morphism $\psi: Z \rightarrow Y$ with $\Phi(\psi)=v$ and $\theta=\varphi \psi$.

This is illustrated as follows.

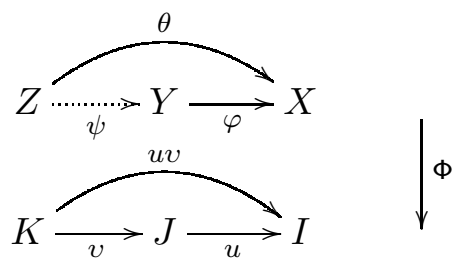

A morphism $\alpha: Z \rightarrow Y$ is called vertical (with respect to $\Phi$ ) if and only if $\Phi(\alpha)$ is an identity isomorphism in $\mathrm{B}$. In particular, for $I \in \mathrm{B}$ we write $\mathrm{X}_{I}$, called the fibre over $I$, for the subcategory of $\mathbf{X}$ consisting of those morphisms $\alpha$ with $\Phi(\alpha)=i d_{I}$,

Definition 1.2 The functor $\Phi: \mathrm{X} \rightarrow \mathrm{B}$ is fibration or category fibred over $\mathrm{B}$ if and only if for all $u: J \rightarrow I$ in $\mathrm{B}$ and $X \in \mathrm{X}_{I}$ there is a cartesian morphism $\varphi: Y \rightarrow X$ over $u$ : such a $\varphi$ is called a cartesian lifting of $X$ along $u$.

If $\Phi: \mathrm{X} \rightarrow \mathrm{B}$ is a fibration, then using the axiom of choice for classes we may select for every $u: J \rightarrow I$ in $\mathrm{B}$ and $X \in \mathrm{X}_{I}$ a cartesian lifting of $\mathrm{X}$ along $\mathrm{u}$

$$
u_{X}: u^{*} X \rightarrow X
$$

Such a choice of cartesian lifting is called a cleavage or splitting of $\Phi$. If we fix the morphism $u: J \rightarrow I$ in $\mathrm{B}$, the splitting gives a so-called reindexing functor

$$
u^{*}: \mathrm{X}_{I} \rightarrow \mathrm{X}_{J}
$$

defined on objects by $X \mapsto u^{*} X$. We can use this re-indexing functor to get an adjoint situation for each $u: J \rightarrow I$ in B. The functor $u^{*}$ is said to give the objects pullback by $u$.

Definition 1.3 Let $\Phi: \mathrm{X} \rightarrow \mathrm{B}$ be a functor. A morphism $\psi: Z \rightarrow Y$ in $\mathrm{X}$ over $v:=\Phi(\psi)$ is called cocartesian if and only if for all $u: J \rightarrow I$ in $\mathrm{B}$ and $\theta: Z \rightarrow X$ with $\Phi(\theta)=u v$ there is a unique morphism $\varphi: Y \rightarrow X$ with $\Phi(\varphi)=u$ and $\theta=\varphi \psi$. 
This is illustrated as follows.

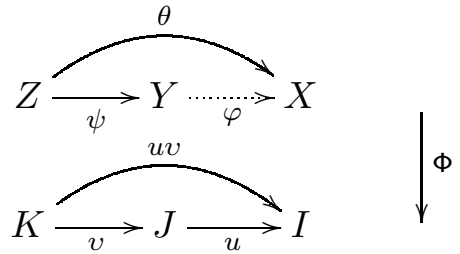

Proposition 1.4 ([8], Proposition 3.7) Let $\Phi: \mathrm{X} \rightarrow \mathrm{B}$ be a fibration of categories. Then $\psi: Z \rightarrow Y$ in $\mathrm{X}$ over $v: K \rightarrow J$ in $\mathrm{B}$ is cocartesian if and only if for all $\theta^{\prime}: Z \rightarrow X^{\prime}$ over $v$ there is a unique morphism $\psi^{\prime}: Y \rightarrow X^{\prime}$ in $\mathrm{X}_{J}$ with $\theta^{\prime}=\psi^{\prime} \psi$.

The functor $\Phi: \mathrm{X} \rightarrow \mathrm{B}$ is cofibration or category cofibred over $\mathrm{B}$ if and only if for all $v: K \rightarrow J$ in $\mathrm{B}$ and $Z \in \mathrm{X}_{K}$ there is a cocartesian morphism $\psi: Z \rightarrow Z^{\prime}$ over $v:$ such a $\psi$ is called a cocartesian lifting of $X$ along $v$.

If $\Phi: \mathrm{X} \rightarrow \mathrm{B}$ is a cofibration, for every morphism $v: K \rightarrow J$ in $\mathrm{B}$ and an object $Z \in \mathrm{X}_{K}$, a cocartesian lifting of $Z$

$$
v_{Z}: Z \rightarrow v_{*}(Z)
$$

along $v$ can be selected. Under these conditions, the functor $v_{*}$ is said to give the objects induced by $v$.

Proposition 1.5 Let $\Phi: \mathrm{X} \rightarrow \mathrm{B}$ be a functor that has a left adjoint $D$. Then for each $K \in \mathrm{ObB}, D(K)$ is initial in $\mathrm{X}_{K}$. In fact if $v: K \rightarrow J$ in $\mathrm{B}$, then for any $X \in \mathrm{X}_{J}$ there is a unique morphism $\epsilon_{K}: D K \rightarrow X$ over $v$.

Theorem 1.6 (8], Theorem 4.2) Let $\Phi: \mathrm{X} \rightarrow \mathrm{B}$ be a fibration of categories which has a left adjoint D. Suppose that $\mathrm{X}$ admits pushouts. Let $v: K \rightarrow J$ be a morphism in $\mathrm{B}$, and let $Z \in \mathbf{X}_{K}$. Then a cocartesian lifting $\psi: Z \rightarrow Y$ of $v$ is given precisely by the pushout in $\mathbf{X}$ :

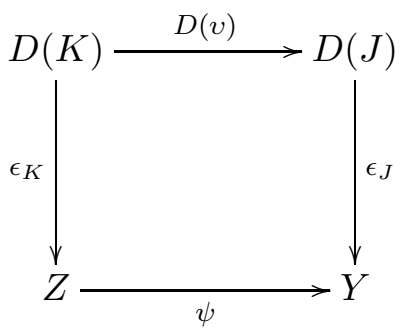

\section{Nil(2)-Modules Fibred and Cofibred Over Groups}

Throughout this paper all actions will be right. The left actions in some references will be rewritten by using right actions. 
Recall from [19] that a pre-crossed module is a group homomorphism $\partial: M \rightarrow Q$ together with an action of $Q$ on $M$, written $m^{q}$ for $q \in Q$ and $m \in M$, satisfying the condition $\partial\left(m^{q}\right)=q^{-1} \partial(m) q$ for all $m \in M$ and $q \in Q$.

$A$ nil(2)-module (cf. [4) is a pre-crossed module $\partial: M \rightarrow Q$ with an additional 'nilpotency' condition. This condition is $P_{3}(\partial)=1$, where $P_{3}(\partial)$ is the subgroup of $M$ generated by Peiffer commutators $\left\langle x_{1}, x_{2}, x_{3}\right\rangle$ of length 3 for $x_{i} \in M$. The Peiffer commutator in a pre-crossed module $\partial: M \rightarrow Q$ is defined by

$$
\langle x, y\rangle=x^{-1} y^{-1} x(y)^{\partial x}
$$

for $x, y \in M$. For a pre-crossed module $\partial: M \rightarrow Q$, if $\langle M, M\rangle=1$, then it is called a crossed module introduced by Whitehead (cf. [21]).

A morphism $(g, f):(M \rightarrow Q) \longrightarrow\left(M^{\prime} \rightarrow Q^{\prime}\right)$ between nil(2)-modules is a pair of homomorphisms of groups $g: M \rightarrow M^{\prime}$ and $f: Q \rightarrow Q^{\prime}$ such that $f \partial=\partial^{\prime} g$ and the actions are preserved, i.e. $g\left(m^{q}\right)=g(m)^{f(q)}$ for any $m \in M, q \in Q$. This defines the category $\operatorname{Nil}(2)$ having nil(2)-modules as objects.

We have a forgetful functor from the category of nil(2)-modules to the category of groups

$$
\Phi_{1}: \operatorname{Nil}(2) \rightarrow \operatorname{Grp}
$$

which sends a nil(2)-module $(M \rightarrow Q)$ to the group $Q$.

This functor has also a left adjoint functor

$$
D_{1}: \operatorname{Grp} \rightarrow \operatorname{Nil}(2)
$$

assigns to a group $Q$ the trivial nil(2)-module $\{1\} \rightarrow Q$.

The following result in the cases of crossed modules of groups and groupoids appeared in [5] and [8] respectively, described in terms of the crossed module of group(oid)s $\sigma_{*} M \rightarrow Q$ induced from the crossed module of group(oid)s $M \rightarrow P$ by a morphism $\sigma: P \rightarrow Q$ in the category of group(oid)s. A similar result for nil(2)-modules over groups can be given as follows:

Proposition 2.1 The forgetful functor $\Phi_{1}: \operatorname{Nil}(2) \rightarrow$ Grp is fibred and cofibred.

Proof: We give the pullback construction of nil(2)-modules to prove that $\Phi_{1}$ is fibred. Suppose that $\partial: M \rightarrow Q$ is a nil(2)-module and $\sigma: P \rightarrow Q$ is a homomorphism of groups. Define $\sigma^{*}(M)$ to be the subgroup of $P \times M$ of elements $(p, m)$ such that $\sigma(p)=\partial(m)$. Let $\sigma_{1}:(p, m) \mapsto m$ and $\beta_{1}:(p, m) \mapsto p$. We obtain the following diagram

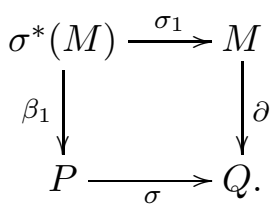


The action of $p^{\prime} \in P$ on $(p, m) \in \sigma^{*}(M)$ can be given by $(p, m)^{p^{\prime}}=\left(p^{\prime-1} p p^{\prime}, m^{\sigma\left(p^{\prime}\right)}\right)$ and $\beta_{1}$ becomes a nil(2)-module. Thus we have a nil(2)-module with the base $P$. This nil(2)-module $\beta_{1}$ is called the pullback of nil(2)-module $\partial$ along $\sigma$. In the above diagram, $\left(\sigma_{1}, \sigma\right)$ becomes cartesian morphism in the category of nil(2)-modules over the morphism $\Phi_{1}\left(\sigma_{1}, \sigma\right)=\sigma$. Therefore the forgetful functor $\Phi_{1}$ is a fibration of categories.

By direct construction, we prove that $\Phi_{1}$ is cofibred. To get it, we give the induced construction of nil(2)-modules.

Let $\mu: M \rightarrow P$ be a nil(2)-module and let $f: P \rightarrow Q$ be a homomorphism of groups. We define $f_{*}(M)=F(M \times Q)$ to be the free group on the set of elements $(m, q)$. The action of $q^{\prime}$ on $(m, q)$ is $(m, q)^{q^{\prime}}=\left(m, q q^{\prime}\right)$. We define as usual $\partial^{\prime}: F(M \times Q) \rightarrow Q$ to be $(m, q) \mapsto q^{-1} f(\mu m) q$. It is well known from [7] that this gives a pre-crossed module over $F(M \times Q) \rightarrow Q$ with a map $i: M \rightarrow F(M \times Q)$ given by $m \mapsto(m, 1)$.

To make $i: M \rightarrow F(M \times Q)$ an operator morphism, we need factor $F(M \times Q)$ out by the relations $(m, q)\left(m^{\prime}, q\right)=\left(m m^{\prime}, q\right)$ and $\left(m^{p}, q\right)=(m, f(p) q)$ for $p \in P$. To make $\partial^{\prime}: F(M \times Q) \rightarrow Q$ a nil(2)-module involves factoring out triple Peiffer elements.

On generators, we have

$$
\theta\left(m^{p}\right)=\left(m^{p}, 1\right)=(m, f(p) 1)=((m, 1))^{f(p)}=\theta(m)^{f(p)}
$$

and $\partial^{\prime} \theta(m)=\partial^{\prime}((m, 1))=f \mu(m)$, then we have the following diagram

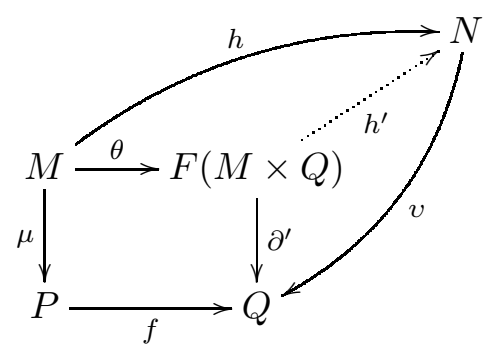

in which $(h, f):(M \rightarrow P) \rightarrow(N \rightarrow Q)$ is a nil(2)-module morphism and $h^{\prime}: F(M \times Q) \rightarrow N$ given by $(m, q) \mapsto h(m)^{q}$ is the necessary unique morphism in $\operatorname{Nil}(2)_{Q}$ which is the category of nil(2)-modules over the same group $Q$, and where $\theta$ is induced from the map $i$. Using Proposition 1.4, we get a nil(2)-module morphism $(\theta, f):(M, P) \rightarrow(F(M \times Q), Q)$ which is cocartesian in $\operatorname{Nil}(2)$ over the homomorphism of groups $\Phi_{1}(\theta, f)=f$. Thus $\Phi_{1}$ is also a cofibration.

\section{Quadratic Modules Fibred over Nil(2)-Modules}

Quadratic modules were introduced by Baues in [4] as algebraic models for topological 3-types (i.e. homotopy connected 3-types). 
Definition 3.1 A quadratic module $(\omega, \delta, \partial)$ is defined as a diagram of $N$-groups

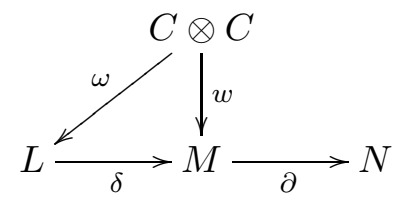

where $\partial$ is a nil(2)-module, $q: M \rightarrow\left(M^{c r}\right)^{a b}=C$ is the natural projection map, $C \otimes C$ has the diagonal action, and the following equalities hold

QM1. $\partial \delta=1$,

QM2. $\delta \omega(q(x) \otimes q(y))=w(q(x) \otimes q(y))=\langle x, y\rangle$,

QM3. $\omega(q(\delta a) \otimes q(x))(q(x) \otimes q(\delta a))=a^{-1} a^{\partial x}$,

QM4. $\omega(q(\delta a) \otimes q(\delta b))=[a, b]$,

for $a, b \in L, x, y \in M$. The natural projection map $q: M \rightarrow\left(M^{c r}\right)^{a b}=C$ is denoted on elements by $x \mapsto q(x)=\{x\}$ for $x \in M$.

A map $\varphi:(\omega, \delta, \partial) \rightarrow\left(\omega^{\prime}, \delta^{\prime}, \partial^{\prime}\right)$ between quadratic modules is given by a commutative diagram, $\varphi=(l, m, n)$

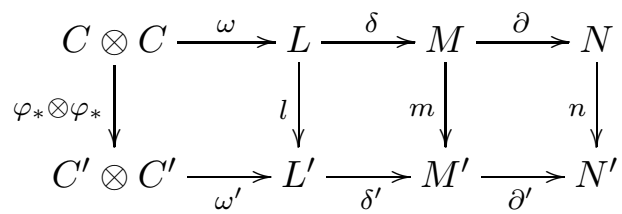

where $(m, n)$ is a morphism between pre-crossed modules which induces $\varphi_{*}: C \rightarrow C^{\prime}$ and where $l$ is an $n$-equivariant homomorphism. Let Quad be the category of quadratic modules and of maps as in the above diagram.

There is a forgetful functor

$$
\Phi: \text { Quad } \rightarrow \operatorname{Nil}(2)
$$

from the category of quadratic modules to the category of nil(2)-modules which sends a quadratic module

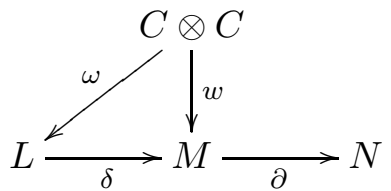

to its base nil(2)-module $(M \stackrel{\partial}{\longrightarrow} N)$.

The left adjoint of this functor is defined as follows. Recall from [4] that any nil(2)-module $\partial: M \rightarrow N$ yields a quadratic module $\bar{\partial}:(1, w, \partial)$ given by

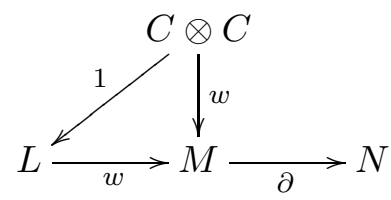


where $L=C \otimes C$. This quadratic module is called the quadratic module associated to the nil(2)-module $\partial$. The category of nil(2)-modules can be considered to be a full subcategory of the category of quadratic modules. It is a reflective subcategory since there is a reflection functor given by Baues in [4]. This is of course functorial and we can say that there is a functor from the category of nil(2)-modules to that of quadratic modules. We denote it by

$$
D: \operatorname{Nil}(2) \rightarrow \text { Quad. }
$$

Therefore, the left adjoint of $\Phi$ assigns to a nil(2)-module $\partial: M \rightarrow N$ the quadratic module written

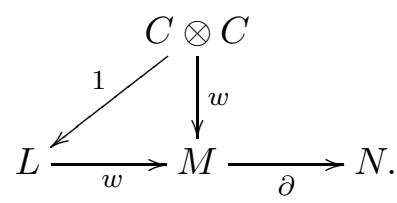

Our first main result is:

Proposition 3.2 The forgetful functor $\Phi:$ Quad $\rightarrow \operatorname{Nil}(2)$ is fibred and has a left adjoint.

Proof: The left adjoint was given above. To prove that $\Phi$ is fibred, we give the pullback construction of a quadratic module.

Consider the quadratic module

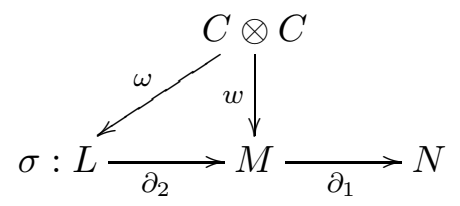

with a morphism of nil(2)-modules $u:=\left(u_{1}, u_{0}\right)$ given by the following commutative diagram:

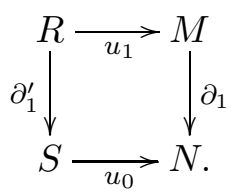

We shall construct a quadratic module on the nil(2)-module $\partial_{1}^{\prime}: R \rightarrow S$.

Let

$$
u^{*}(L)=\left\{(r, l): r \in \operatorname{ker} \partial_{1}^{\prime}, u_{1}(r)=\partial_{2}(l)\right\} \subset R \times L .
$$

We have a commutative diagram

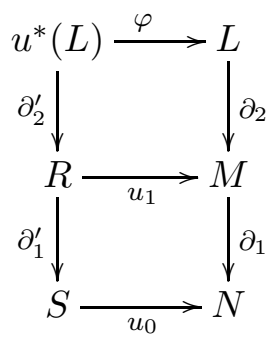


in which $\varphi(r, l)=l$ and $\partial_{2}^{\prime}(r, l)=r$ for $(r, l) \in u^{*}(L)$.

Let $C^{\prime}=\left(R^{c r}\right)^{a b}$. We can define the quadratic map $\omega^{\prime}$ from $C^{\prime} \otimes C^{\prime}$ to $L$ as

$$
\omega^{\prime}\left(\{r\} \otimes\left\{r^{\prime}\right\}\right)=\left(\left\langle r, r^{\prime}\right\rangle, \omega\left\{u_{1}(r)\right\} \otimes\left\{u_{1}\left(r^{\prime}\right)\right\}\right)
$$

where $\omega$ is the quadratic map of the quadratic module $\sigma$. The action of $S$ on $u^{*}(L)$ is given by $(r, l)^{s}=\left(r^{s}, l^{u_{0}(s)}\right)$ for $s \in S,(r, l) \in u^{*}(L)$. Then since $u_{1}\left(r^{s}\right)=u_{1}(r)^{u_{0}(s)}=$ $\partial_{2}(l)^{u_{0}(s)}=\partial_{2}\left(l^{u_{0}(s)}\right)$ and $\partial_{1}^{\prime}$ is a pre-crossed module, for $r \in \operatorname{ker} \partial_{1}^{\prime}$ we have $r^{s} \in \operatorname{ker} \partial_{1}^{\prime}$ and $\left(r^{s}, l^{u_{0}(s)}\right) \in u^{*}(L)$.

We obtain that the diagram of homomorphisms of groups

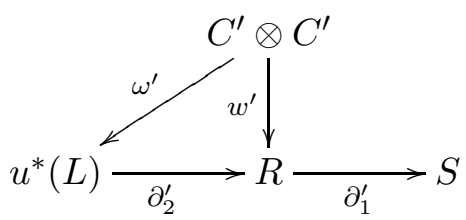

is a quadratic module. (See $\left[\bullet^{1}\right]$ in Appendix)

It can be easily shown that $\left(\varphi, u_{1}, u_{0}\right)$ is a morphism between quadratic modules. Now we show that the morphism $\left(\varphi, u_{1}, u_{0}\right)$ is a cartesian morphism in the category of quadratic modules over the morphism $\Phi\left(\varphi, u_{1}, u_{0}\right)=\left(u_{1}, u_{0}\right)$. Let $\left(v_{1}, v_{0}\right): \partial_{1}^{\prime \prime} \rightarrow \partial_{1}^{\prime}$ be morphism between nil(2)-modules given by the commutative diagram

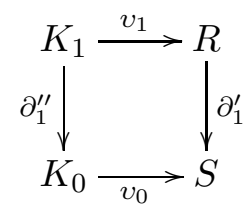

and

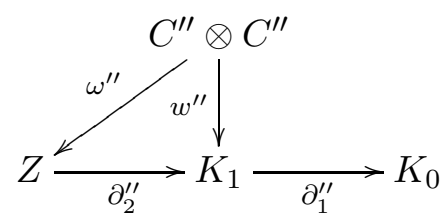

be quadratic module with a morphism $\left(\theta, u_{1}^{\prime}, u_{0}^{\prime}\right)$ from the quadratic module $\left(\omega^{\prime \prime}, \partial_{2}^{\prime \prime}, \partial_{1}^{\prime \prime}\right)$ to the quadratic module $\left(\omega, \partial_{2}, \partial_{1}\right)$ together with $u_{1} v_{1}=u_{1}^{\prime}$ and $u_{0} v_{0}=u_{0}^{\prime}$.

We have the following commutative diagram

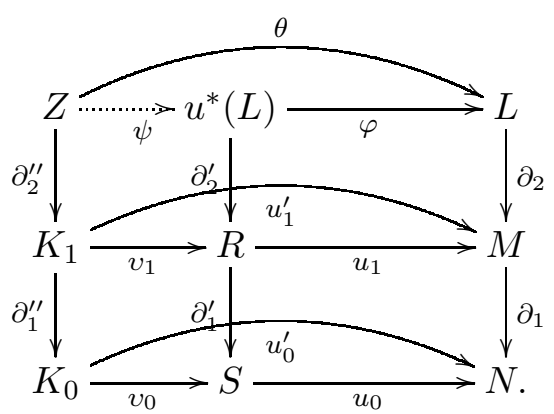


The necessary unique quadratic module morphism $\left(\psi, v_{1}, v_{0}\right)$ is given by $\psi(z)=\left(v_{1} \partial_{2}^{\prime \prime}(z), \theta(z)\right)$, for $z \in Z$. We show $\left[\bullet^{2}\right]$ in Appendix that $\left(\varphi, u_{1}, u_{0}\right)$ is a morphism of quadratic modules.

Then the morphism $\left(\varphi, u_{1}, u_{0}\right)$ is a cartesian morphism in Quad over the morphism $\Phi\left(\varphi, u_{1}, u_{0}\right)=u:=\left(u_{1}, u_{0}\right)$. The quadratic module

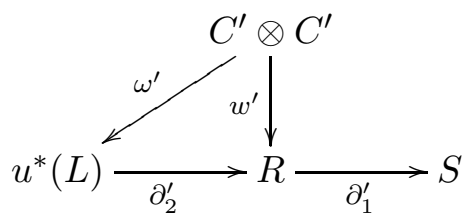

is called the pullback of the quadratic module

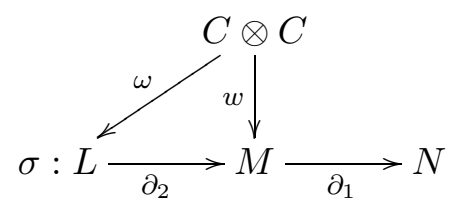

by the morphism $u:=\left(u_{1}, u_{0}\right)$ between nil(2)-modules.

We may select a cartesian lifting of $\sigma$ along $u:=\left(u_{1}, u_{0}\right)$

$$
u^{\sigma}: u^{*}(\sigma) \rightarrow \sigma
$$

where $u^{*}(\sigma)$ is the pullback quadratic module

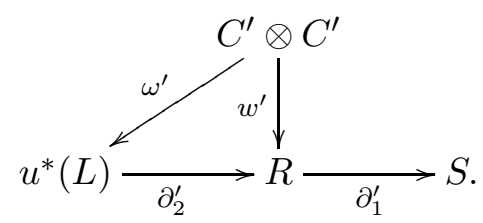

If we fix the morphism $u:=\left(u_{1}, u_{0}\right)$ from $\left(\partial_{1}^{\prime}: R \rightarrow S\right)$ to $\left(\partial_{1}: M \rightarrow N\right)$ in the category of nil(2)-modules, we obtain a re-indexing functor

$$
u^{*}: \operatorname{Quad}_{(M \rightarrow N)} \longrightarrow \operatorname{Quad}_{(R \rightarrow S)}
$$

defined on objects by $\sigma \mapsto u^{*}(\sigma)$ and where Quad $(M \rightarrow N)$ denotes the category of quadratic modules over the same nil(2)-module $\partial_{1}: M \rightarrow N$. In particular there is, for a fixed nil(2)module $\partial: M \rightarrow N$, a subcategory $\operatorname{Quad}_{(M \rightarrow N)}$ of Quad which has as objects those quadratic modules with $\partial: M \rightarrow N$ as the 'base'.

Using Proposition 2.5 of [8], for this re-indexing functor $u^{*}$, there is a bijection

$$
\operatorname{Quad}_{(R \rightarrow S)}\left(\kappa, u^{*}(\sigma)\right) \cong \operatorname{Quad}_{\left(u_{1}, u_{0}\right)}(\kappa, \sigma)
$$

natural in $\kappa \in \operatorname{Quad}_{(R \rightarrow S)}, \sigma \in \operatorname{Quad}_{(M \rightarrow N)}$ and where $\operatorname{Quad}_{\left(u_{1}, u_{0}\right)}$ consists of those morphisms $\left(\alpha, u_{1}, u_{0}\right): \kappa \rightarrow \sigma$ in Quad with $\Phi\left(\alpha, u_{1}, u_{0}\right)=u:=\left(u_{1}, u_{0}\right)$. 


\section{Quadratic Modules Cofibred Over Nil(2)-modules}

We give the construction of cofibration of $\Phi$. Brown and Higgins in [5] described the induced crossed module $u_{*}(M) \rightarrow Q$ from a crossed module $M \rightarrow P$ by a morphism $u: P \rightarrow Q$ in the category of groups. That is, they proved that the forgetful functor from the category of crossed modules to the category of groups which sends $(M \rightarrow P) \mapsto P$ is a fibration and also a cofibration of categories. We will extend this result for the functor

$$
\Phi: \text { Quad } \rightarrow \operatorname{Nil}(2)
$$

This gives that quadratic modules cofibred over nil(2)-modules and the notion of induced quadratic module. By a similar way, the notion of induced 2-crossed module has been constructed in [1].

Proposition 4.1 The forgetful functor $\Phi:$ Quad $\rightarrow \operatorname{Nil}(2)$ is cofibred.

Proof: We prove this by a direct construction. Consider the quadratic module

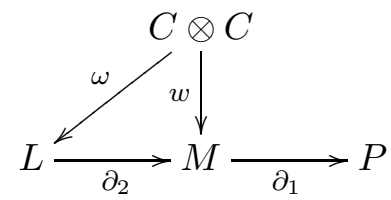

with a morphism of nil(2)-modules $v:\left(v_{1}, v_{0}\right)$ from $(M \rightarrow P)$ to $(N \rightarrow Q)$ given by a commutative diagram

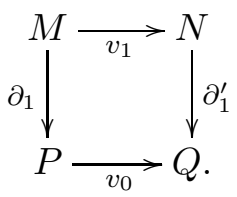

We shall construct a quadratic module on the nil(2)-module $N \rightarrow Q$.

For this, consider the free product

$$
F(N \times L) *\langle C \otimes C\rangle
$$

where $\langle C \otimes C\rangle$ is the free group generated by the elements of the form $\langle\{a\} \otimes\{b\}\rangle$ for $a, b \in N$ and $F(N \times L)$ is the free group generated by the set $N \times L$ and $C=\left(N^{c r}\right)^{a b}$. Observe that action of $Q$ on $N$ induces an action on $\langle C \otimes C\rangle$ by

$$
(\{a\} \otimes\{b\})^{q}=\left(\left\{a^{q}\right\} \otimes\left\{b^{q}\right\}\right)
$$

and induces an action on $F(N \times L)$ given by $(n, l)^{q}=\left(n^{q}, l\right)$. To get an induced quadratic module, factor $F(N \times L) *\langle C \otimes C\rangle$ out by the relations:

1. $\left(\left\{n^{-1} v_{1} \partial_{2} l n\right\} \otimes\{b\}\right)\left(\{b\} \otimes\left\{n^{-1} v_{1} \partial_{2} l n\right\}\right)=(n, l)^{-1}(n, l)^{\partial_{1}^{\prime} b}$ 
2. $\left(\left\{n^{-1} v_{1} \partial_{2} l n\right\} \otimes\left\{n^{\prime-1} v_{1} \partial_{2} l^{\prime} n^{\prime-1}\right\}=\left[(n, l),\left(n^{\prime}, l^{\prime}\right)\right]\right.$

for $n, n^{\prime}, b \in N, l, l^{\prime} \in L$.

We have a morphism $\partial_{2}^{\prime}: F(N \times L) *\langle C \otimes C\rangle \rightarrow N$ induced on $F(N \times L)$ by

$$
(n, l) \mapsto n^{-1}\left(v_{1} \partial_{2} l\right) n
$$

and given on $\langle C \otimes C\rangle$ by

$$
\langle\{x\} \otimes\{y\}\rangle \mapsto x^{-1} y^{-1} x y^{\partial_{1}^{\prime} x}
$$

for $x, y \in N$. Then, the diagram

$$
F(N \times L) *\langle C \otimes C\rangle \stackrel{\partial_{2}^{\prime}}{\longrightarrow} N \stackrel{\partial_{1}^{\prime}}{\longrightarrow} Q
$$

is a complex of homomorphisms of groups. (See $\left[\bullet^{3}\right]$ Appendix)

We define the quadratic map

$$
\omega^{\prime}: C \otimes C \longrightarrow F(N \times L) *\langle C \otimes C\rangle
$$

by $\omega^{\prime}(\{x\} \otimes\{y\})=\langle\{x\} \otimes\{y\}\rangle$ for $x, y \in N$.

We define $\psi: L \rightarrow F(N \times L) *\langle C \otimes C\rangle$ by $\psi(l)=(1, l)$ in the following diagram,

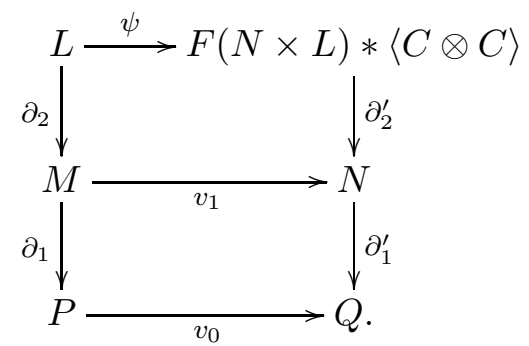

We now wish to change the map $\psi: L \rightarrow F(N \times L) *\langle C \otimes C\rangle$ to make it operator morphism in Quad. For this we need to add in $F(N \times L) *\langle C \otimes C\rangle$ new relations:

1. $(1, \omega\{x\} \otimes\{y\})=\left\langle\left\{v_{1}(x)\right\} \otimes\left\{v_{1}(y)\right\}\right\rangle$

2. $\left(1, l^{p}\right)=\left(v_{0} p, l\right)$

for $p \in P, l \in L$ and $x, y \in M$. Then $\left(\psi, v_{1}, v_{0}\right)$ is a morphism of quadratic modules. (See $[\bullet]$ Appendix)

Now, we show that the morphism $\left(\psi, v_{1}, v_{0}\right)$ in the category of quadratic modules is cocartesian over the morphism $\Phi\left(\psi, v_{1}, v_{0}\right)=v:=\left(v_{1}, v_{0}\right)$ in the category of nil(2)-modules. For this, we use Proposition 1.4 given by Brown and Sivera in 8 ].

Let

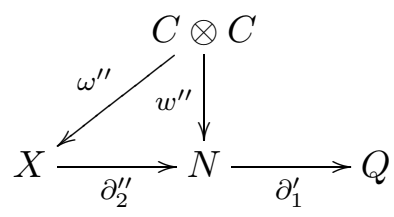


be a quadratic module in Quad $(N \rightarrow Q)$ with a morphism of quadratic modules $\left(\theta^{\prime}, v_{1}, v_{0}\right)$ given by the commutative diagram:

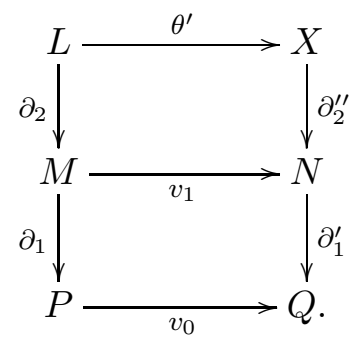

Then, there is a unique morphism $\theta_{*}: F(N \times L) *\langle C \otimes C\rangle \rightarrow X$ such that the diagram

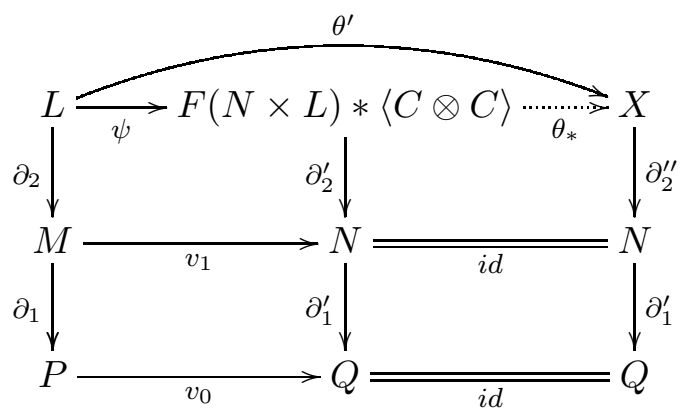

commutes.

The necessary unique morphism $\theta_{*}$ is defined as follows: For generators $(n, l)$ of $F(N \times L)$, $\theta_{*}$ is given by $\theta_{*}(n, l)=\left(\theta^{\prime}(l)\right)^{n}$. For generators $\langle\{x\} \otimes\{y\}\rangle$ of $\langle C \otimes C\rangle, \theta_{*}$ is defined by $\theta_{*}\langle\{x\} \otimes\{y\}\rangle=\omega^{\prime \prime}(\{x\} \otimes\{y\})$ where $\omega^{\prime \prime}$ is the quadratic map from $C \otimes C$ to $X$. (See $\left[\bullet^{5}\right]$ in Appendix) Using Proposition 1.4, the morphism $\left(\psi, v_{1}, v_{0}\right)$ becomes a cocartesian morphism in Quad over $\Phi\left(\psi, v_{1}, v_{0}\right)=v:=\left(v_{1}, v_{0}\right)$. Clearly, the forgetful functor

$$
\Phi: \text { Quad } \rightarrow \operatorname{Nil}(2)
$$

is cofibred.

Thus, together with the relations in $F(N \times L) *\langle C \otimes C\rangle$ given above, the diagram

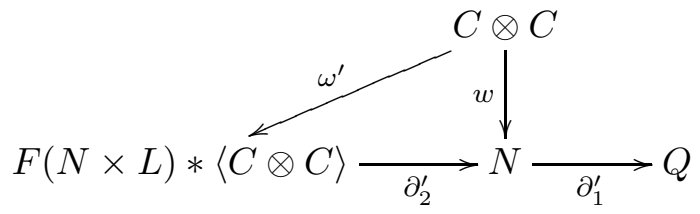

is an induced quadratic module by the morphism $v:=\left(v_{1}, v_{0}\right)$ of nil(2)-modules.

Consequently, for a nil(2)-module morphism

$$
v:=\left(v_{1}, v_{0}\right):\left(M \stackrel{\partial_{1}}{\longrightarrow} P\right) \longrightarrow\left(N \stackrel{\partial_{1}^{\prime}}{\longrightarrow} Q\right)
$$

we obtain a functor

$$
v_{*}: \operatorname{Quad}_{(M \rightarrow P)} \longrightarrow \operatorname{Quad}_{(N \rightarrow Q)}
$$


which sends a quadratic module

$$
L \stackrel{\partial_{2}}{\longrightarrow} M \stackrel{\partial_{1}}{\longrightarrow} P
$$

to the induced quadratic module

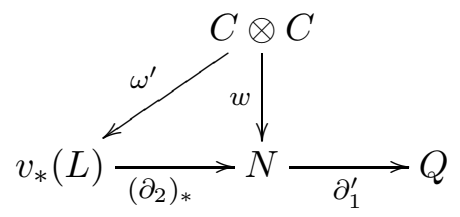

where $v_{*}(L)=F(N \times L) *\langle C \otimes C\rangle$ with the relations and $\left(\partial_{2}\right)_{*}=\partial_{2}^{\prime}$.

Therefore we obtain the following result.

Theorem 4.2 Let

$$
v:=\left(v_{1}, v_{0}\right):\left(M \stackrel{\partial_{1}}{\longrightarrow} P\right) \longrightarrow\left(N \stackrel{\partial_{1}^{\prime}}{\longrightarrow} Q\right)
$$

be a morphism of nil(2)-modules. The functor

$$
v_{*}: \operatorname{Quad}_{(M \rightarrow P)} \longrightarrow \operatorname{Quad}_{(N \rightarrow Q)}
$$

is left adjoint to the pullback functor

$$
v^{*}: \operatorname{Quad}_{(N \rightarrow Q)} \longrightarrow \operatorname{Quad}_{(M \rightarrow P)} .
$$

Proof: Let

$$
L \stackrel{\partial_{2}}{\longrightarrow} M \stackrel{\partial_{1}}{\longrightarrow} P
$$

be a quadratic module. The diagram

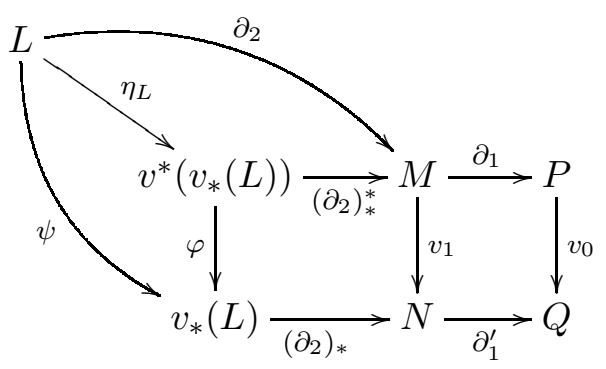

determines the morphism in $\operatorname{Quad}_{(M \rightarrow P)}$

$$
\left(\eta_{L}, i d, i d\right):\left(L \stackrel{\partial_{2}}{\longrightarrow} M \stackrel{\partial_{1}}{\longrightarrow} P\right) \rightarrow\left(v^{*}\left(v_{*} L\right) \stackrel{\left(\partial_{2}\right)_{*}^{*}}{\longrightarrow} M \stackrel{\partial_{1}}{\longrightarrow} P\right) .
$$

We show that this is a universal morphism. Let

$$
X \stackrel{\partial_{2}^{\prime \prime}}{\longrightarrow} N \stackrel{\partial_{1}^{\prime}}{\longrightarrow} Q
$$


be a quadratic module. Given a morphism

$$
(t, i d, i d):\left(L \stackrel{\partial_{2}}{\longrightarrow} M \stackrel{\partial_{1}}{\longrightarrow} P\right) \rightarrow\left(v^{*}(X) \stackrel{\left(\partial_{2}^{\prime \prime}\right)^{*}}{\longrightarrow} M \stackrel{\partial_{1}}{\longrightarrow} P\right)
$$

in $\operatorname{Quad}_{(M \rightarrow P)}$, we consider the composition morphism

$$
\left(\varphi t, v_{1}, v_{0}\right):\left(L \stackrel{\partial_{2}}{\longrightarrow} M \stackrel{\partial_{1}}{\longrightarrow} P\right) \rightarrow\left(X \stackrel{\left(\partial_{2}^{\prime \prime}\right)}{\longrightarrow} N \stackrel{\partial_{1}^{\prime}}{\longrightarrow} Q\right) .
$$

By Proposition 4.1, there exists only one morphism

$$
\left(v_{*}(L) \stackrel{\left(\partial_{2}\right)_{*}}{\longrightarrow} N \stackrel{\partial_{1}^{\prime}}{\longrightarrow} Q\right) \rightarrow\left(X \stackrel{\left(\partial_{2}^{\prime \prime}\right)}{\longrightarrow} N \stackrel{\partial_{1}^{\prime}}{\longrightarrow} Q\right)
$$

in $\operatorname{Quad}_{(N \rightarrow Q)}$, which commutes the following diagram:

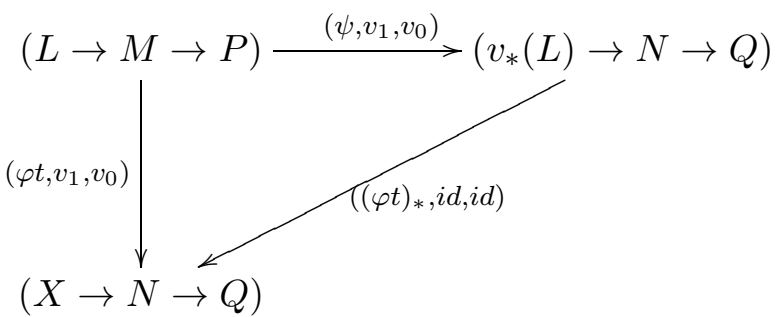

Through the construction of $\eta_{L}$ and $v^{*}$ it is evident that:

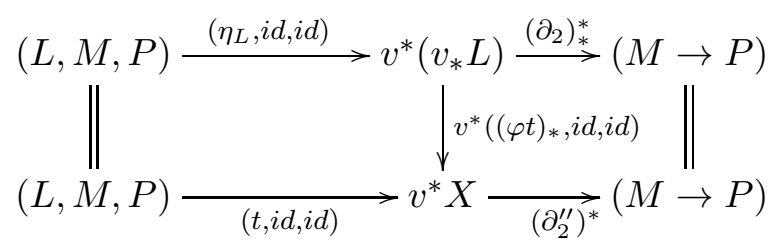

since:

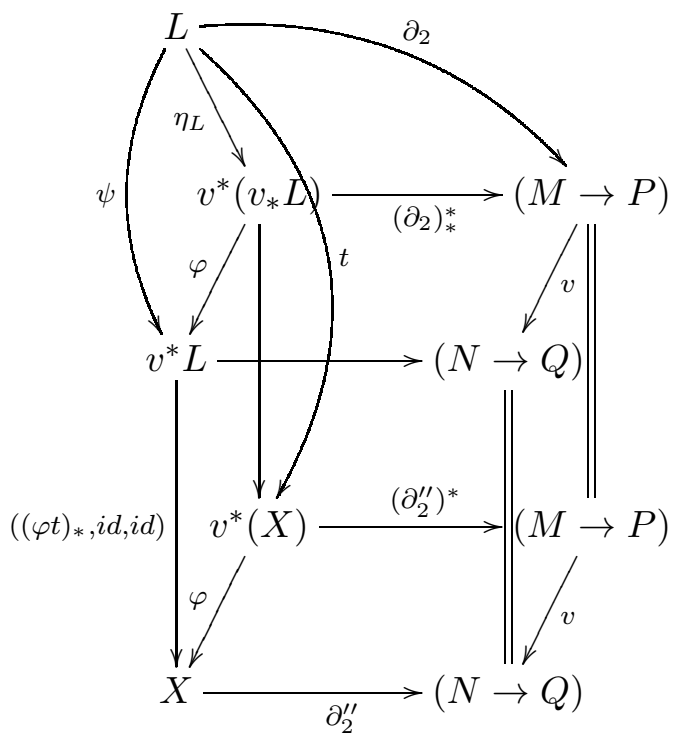

Thus $v_{*}$ is left adjoint to $v^{*}$. 


\subsection{Another Presentation of Induced Quadratic Modules}

Crossed squares were introduced in [15]. Quadratic modules are related to crossed squares, and induced crossed squares have been studied in [7, 14] and also in Appendix B4 of [6]. The following method used in [8] gives another view of a presentation of the induced crossed square, and which is applied to free crossed squares in [14].

In Theorem 1.6, by taking $X=$ Quad and $B=\operatorname{Nil}(2)$, we can give another view of a description of the induced quadratic module.

We know that the functor $\Phi:$ Quad $\rightarrow \operatorname{Nil}(2)$ is a fibration and has a left adjoint

$$
D: \operatorname{Nil}(2) \rightarrow \text { Quad. }
$$

Recall from the previous section that this left adjoint $D$ assigns to a nil(2)-module $\partial: M \rightarrow P$ the quadratic module written

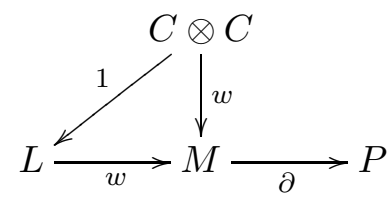

where $L=C \otimes C$. We denote this associated quadratic module by

$$
D(M \rightarrow P)=C \otimes C \stackrel{w}{\longrightarrow} M \longrightarrow P
$$

We know from [4 that the category of quadratic modules admits pushouts. Then $\Phi$ is a fibration of categories and also a cofibration. Thus we have a notion of induced quadratic module, as follows:

Given a quadratic module

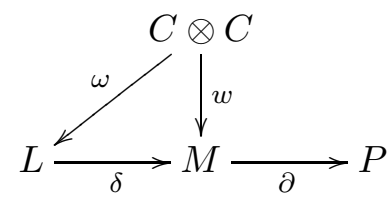

and a morphism of nil(2)-modules

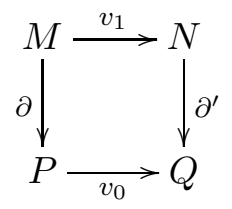

we get an induced quadratic module

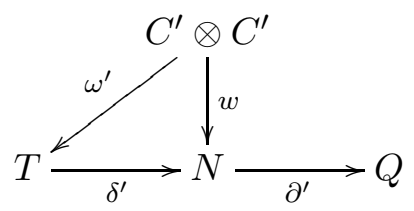


which according to Theorem 1.6 is given by a pushout in the category of quadratic modules of the form

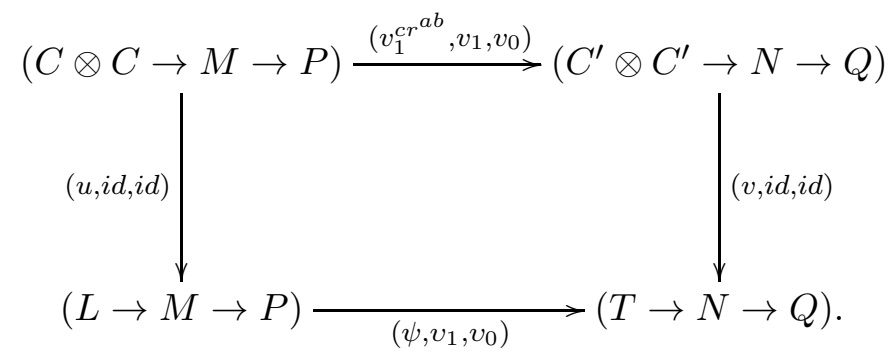

This gives another view of a presentation of the induced quadratic module given above.

\section{$5 \quad$ Applications to Reduced Quadratic Modules}

Recall that a reduced quadratic module is a quadratic module

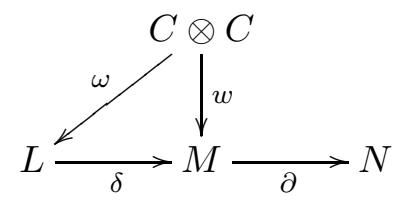

in which the group $\mathrm{N}$ is a trivial group. More clearly, a reduced quadratic module $(\omega, \delta)$ is a diagram

$$
M^{a b} \otimes M^{a b} \stackrel{\omega}{\longrightarrow} L \stackrel{\delta}{\longrightarrow} M
$$

of homomorphisms between groups such that the following conditions hold:

(i) The group $M$ is a nil(2)-group and the quotient map $M \rightarrow M^{a b}$ to the abelianization $M^{a b}$ of $M$ is denoted by $x \mapsto \bar{x}$.

(ii) For $x, y \in M, \delta \omega(\bar{x} \otimes \bar{y})=[x, y]$.

(iii) For $a \in L, x \in M, \omega(\overline{\delta a} \otimes \bar{x})(\bar{x} \otimes \overline{\delta a})=1$.

(iv) For $a, b \in L, \omega(\overline{\delta a} \otimes \overline{\delta b})=[a, b]$.

We denote the category of reduced quadratic modules by RQM. Since in a reduced quadratic module

$$
C \otimes C \stackrel{\omega}{\longrightarrow} L \stackrel{\delta}{\longrightarrow} M
$$

the group $M$ is a nil(2)-group, we have a forgetful functor

$$
\Phi_{R}: \mathrm{RQM} \longrightarrow \operatorname{NilGrp}(2)
$$

from the category of reduced quadratic modules to that of nil(2)-groups which sends

$$
(C \otimes C \stackrel{\omega}{\longrightarrow} L \stackrel{\delta}{\longrightarrow} M) \longrightarrow M .
$$

Using the construction method given in the previous section, we can give the following proposition. 
Proposition 5.1 The functor $\Phi_{R}: \mathrm{RQM} \rightarrow \mathrm{NilGrp}(2)$ is a bifibration.

We now compare the universal properties defining the induced reduced quadratic module and the free reduced quadratic module on a map. Using the induced reduced quadratic module constructed above, we get an alternative description of the free reduced quadratic module.

\section{Free Reduced Quadratic Modules}

In [2], using the suspension functor given in [17], the construction of a free reduced quadratic module was given. We recall the definition of free reduced quadratic modules. Let

$$
C \otimes C \stackrel{\omega}{\longrightarrow} L \stackrel{\delta}{\longrightarrow} M
$$

be a reduced quadratic module, $Y$ be a set and $v: Y \rightarrow L$ be a function. Then this reduced quadratic module is said to be a free reduced quadratic module on function $v: Y \rightarrow L$ if for any reduced quadratic module

$$
C^{\prime} \otimes C^{\prime} \stackrel{\bar{\omega}}{\longrightarrow} L^{\prime} \stackrel{\bar{\delta}}{\longrightarrow} M
$$

and a function $v^{\prime}: Y \rightarrow L^{\prime}$ such that $\delta v=\bar{\delta} v^{\prime}$, there is a unique morphism $\Phi: L \rightarrow L^{\prime}$ such that $\bar{\delta} \Phi=\delta$. This situation can be illustrated as follows:

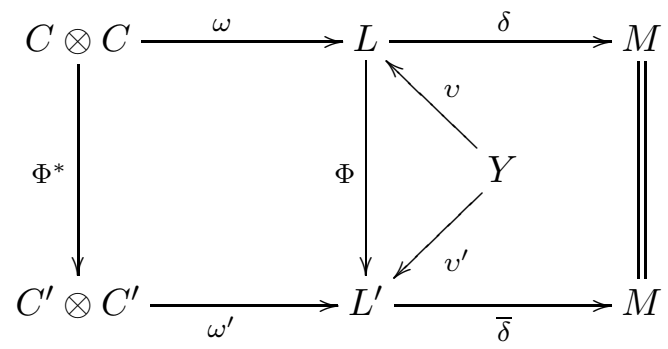

Proposition 5.2 Let $P$ be a nil(2)-group and $\left\{\theta_{r}: r \in R\right\}$ be an indexed family of elements of $P$, or equivalently, a map $\theta: R \rightarrow P$. Let $F$ be the free group generated by $R$ and $F^{\text {nil }}$ be the nilization of $F$ with the quotient map $g: F \rightarrow F^{n i l}, r \mapsto \bar{r}$. Let $f^{n i l}: F^{\text {nil }} \rightarrow P$ be the homomorphism of groups such that $f^{n i l}(\bar{r})=\theta_{r} \in P$. We have the following reduced quadratic module

$$
(\omega, 1)=C \otimes C \stackrel{\omega}{\longrightarrow} F^{n i l} \stackrel{1_{F^{n i l}}}{\longrightarrow} F^{n i l}
$$

where $\omega$ is given by $\omega\{\bar{r}\} \otimes\left\{\overline{r^{\prime}}\right\}=\left[\bar{r}, \overline{r^{\prime}}\right]$ and where $\bar{r} \in F^{n i l},\{\bar{r}\} \in C$. Then the reduced quadratic module

$$
C^{\prime} \otimes C^{\prime} \longrightarrow F\left(P \times F^{n i l}\right) *\left\langle C^{\prime} \otimes C^{\prime}\right\rangle=f_{*}{ }^{n i l}(P) \stackrel{\bar{\delta}}{\longrightarrow} P
$$

induced from $(\omega, 1)$ by $f^{\text {nil }}$ is the free reduced quadratic module on $\{(1, \bar{r}): r \in R\}$. 
Proof: First, consider the following diagram

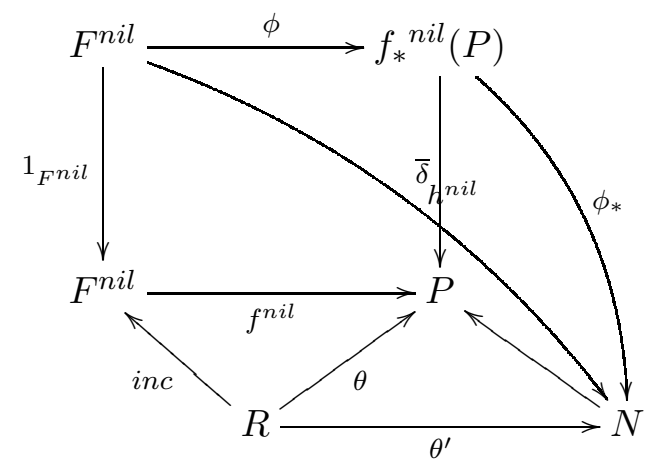

in which $\phi(\bar{r})=(1, \bar{r})$. We shall check that the data in the free and induced constructions are equivalent. The data in the induced construction are a reduced quadratic module

$$
\left(\omega^{\prime \prime}, \partial\right): C^{\prime} \otimes C^{\prime} \stackrel{\omega^{\prime \prime}}{\longrightarrow} N \stackrel{\partial}{\longrightarrow} P
$$

and a morphism of $\left(h^{n i l}, f^{n i l}\right):\left(\omega^{\prime}, 1\right) \rightarrow\left(\omega^{\prime \prime}, \partial\right)$. The data in the free reduced quadratic module construction are a reduced quadratic module

$$
\left(\omega^{\prime \prime}, \partial\right): C^{\prime} \otimes C^{\prime} \stackrel{\omega^{\prime \prime}}{\longrightarrow} N \stackrel{\partial}{\longrightarrow} P
$$

and a map $\theta^{\prime}: R \rightarrow N$ with $\partial \theta^{\prime}=\theta$. Since $F$ is the free group on $R$, the map $\theta^{\prime}$ is equivalent to a homomorphism $h: F \rightarrow N$ lifting $\theta$ (i.e. $h(r)=\theta^{\prime}(r)$ ). We have a morphism from $h$, $h^{\text {nil }}: F^{n i l} \rightarrow N$ by $\left.h^{n i l}(\bar{r})=h(r)=\theta^{\prime}(r)\right)$. Moreover $h^{\text {nil }}$ satisfies

$$
\begin{aligned}
h^{n i l} \omega\{\bar{r}\} \otimes\left\{\overline{r^{\prime}}\right\} & =h^{n i l}\left[\bar{r}, \overline{r^{\prime}}\right] \\
& =h^{n i l}\left(\left[\bar{r}, \overline{r^{\prime}}\right]\right) \\
& =\left[\theta^{\prime}(r), \theta^{\prime}\left(r^{\prime}\right)\right] \\
& =\omega^{\prime \prime}\left\{\partial \theta^{\prime} r\right\} \otimes\left\{\partial \theta^{\prime} r^{\prime}\right\} \\
& =\omega^{\prime \prime}\left\{\theta_{r}\right\} \otimes\left\{\theta_{r^{\prime}}\right\} \\
& =\omega^{\prime \prime}\left\{f^{n i l}(\bar{r})\right\} \otimes\left\{f^{n i l}\left(\overline{r^{\prime}}\right)\right\}
\end{aligned}
$$

for all $r, r^{\prime} \in R$. So $\left(h^{n i l}, f^{n i l}\right)$ is a morphism of reduced quadratic modules. Thus the data in both cases are equivalent.

Now, we develop an example of a finite reduced quadratic module to see what it looks like and how the induced construction behave on it. First, we give the following result.

Proposition 5.3 Let $\phi: M \rightarrow N$ be a monomorphism of groups and let

$$
C \otimes C \stackrel{\omega}{\longrightarrow} L \stackrel{\partial}{\longrightarrow} M
$$

be a reduced quadratic module and $T$ be a right transversal of $\phi(M)$ in $N$ and $(L * T) *\left\langle C^{\prime} \otimes C^{\prime}\right\rangle$ be the free product of $(L \vec{*} T)$ and $\left\langle C^{\prime} \otimes C^{\prime}\right\rangle$ where $(L \vec{*} T)$ is the free product of groups $L_{t}, t \in T$, 
with elements $(l, t), l \in L$ isomorphic to $L$ under the map $(l, t) \rightarrow l$ and $\left\langle C^{\prime} \otimes C^{\prime}\right\rangle$ is the free group generated by the set $C^{\prime} \otimes C^{\prime}$. If $n \in N$ acts on $(L \vec{*} T)$ by the rule ${ }^{n}(l, t)={ }^{u}(l, m)$ where $m \in M, u \in T, n t=u \phi(m)$, then

$$
C^{\prime} \otimes C^{\prime} \stackrel{\omega}{\longrightarrow} \phi_{*}(L)=(L \vec{*} T) *\left\langle C^{\prime} \otimes C^{\prime}\right\rangle / S \stackrel{\beta}{\longrightarrow} Q
$$

is a reduced quadratic module with the map $\omega(\bar{a} \otimes \bar{b})=\langle\bar{a} \otimes \bar{b}\rangle S$ for $\bar{a}, \bar{b} \in N^{a b}$, where $S$ is the normal closure in $\phi_{*}(L)$ of elements

$$
\begin{gathered}
\left\langle\overline{\beta(l, t)} \otimes \overline{\beta\left(l^{\prime}, t^{\prime}\right)}\right\rangle=\left[(l, t),\left(l^{\prime}, t^{\prime}\right)\right] \\
\langle\overline{\beta(l, t)} \otimes \bar{n}\rangle\langle\bar{n} \otimes \overline{\beta(l, t)}\rangle=1 \\
\omega\{m\} \otimes\left\{m^{\prime}\right\}=\left\langle\overline{\phi(m)} \otimes \overline{\phi\left(m^{\prime}\right)}\right\rangle
\end{gathered}
$$

for $l \in L, t \in T, m, m^{\prime} \in M, n \in N$.

Example 5.4 (The Dihedral Reduced Quadratic Module) Let

$$
P^{a b} \otimes P^{a b} \stackrel{\omega}{\longrightarrow} M \stackrel{\partial}{\longrightarrow} P
$$

be a reduced quadratic module and $i: P \rightarrow Q$ be a homomorphism. Suppose that $Q=D_{4}$ with the presentation $\left\langle a, b: a^{4}=b^{2}=a b a b=1\right\rangle$ is the dihedral group of order 8 . Let $M=P$ be the cyclic subgroup of $D_{4}$ of order 2 generated by $b$. Since $Z\left(D_{4}\right)=\left\{1, a^{2}\right\}$, we have $D_{4} / Z\left(D_{4}\right) \cong$ Klein $4-$ group $=C$. Since $C$ is an Abelian group, the dihedral group $D_{4}$ is a nil(2)-group. Let $C_{4}=\{0,1,2,3\}$ be the cyclic group of order 4. A right transversal $T$ of $C_{2}=\{1, b\}$ in $D_{4}$ is given by the elements $1, x, x^{2}, x^{3}$, since $D_{4} / C_{2}=\left\{C_{2}, a C_{2}, a^{2} C_{2}, a^{3} C_{2}\right\}$. We have $i_{*}\left(C_{2}\right)=\left(C_{2} \vec{*} T\right) *\langle C \otimes C\rangle$ where $i: C_{2} \rightarrow D_{4}$ is the inclusion map and $C=\left(D_{4}\right)^{a b}$ is Klein 4-group. Hence the generators of $\left(C_{2} \vec{*} T\right)$ are in the forms $r_{i}=\left(b, a^{i}\right)$ with the relations $\left(r_{i}\right)^{2}=1, i=0,1,2,3$ and $\langle C \otimes C\rangle$ is the free group generated by elements of the forms $\left\langle b a^{i} \otimes b a^{j}\right\rangle, i, j=0,1,2$ with the relations

$$
\begin{aligned}
& \text { 1. }\left\langle b a^{2 i} \otimes b a^{2 j}\right\rangle=a^{2 i+2 j} \in\left\langle a^{2}\right\rangle \\
& \text { 2. }\left\langle b a^{2 i} \otimes b a^{j}\right\rangle\left\langle b a^{j} \otimes b a^{2 i}\right\rangle=1 .
\end{aligned}
$$

We have a morphism

$$
\delta:\left(C_{2} \vec{*} T\right) *\langle C \otimes C\rangle \longrightarrow D_{4}
$$

defined on generators $\left(b, a^{i}\right)$ by $\delta\left(b, a^{i}\right)=b a^{2 i}$ and on generators $\left\langle b a^{i} \otimes b a^{j}\right\rangle$ by

$$
\delta\left(\left\langle b a^{i} \otimes b a^{j}\right\rangle\right)=a^{2 i+2 j} .
$$

Thus we have

$$
\begin{array}{cl}
\delta\left(b \otimes a^{2}\right)=a^{4}=1=\delta\left(a^{2} \otimes b\right), & \delta(b \otimes a)=a^{2}=[b, a]=[a, b]=\delta(a \otimes b), \\
\delta(b \otimes b)=1=[b, b], & \delta\left(a^{2} b \otimes a\right)=1=\left[a^{2}, a\right], \\
\delta(b a \otimes b)=[b a, b]=a^{2}, & \delta(b a \otimes b a)=a^{4}=1=[b a, b a], \\
\delta(b \otimes b a)=a^{2}=[b, b a] . &
\end{array}
$$


Thus we have a reduced quadratic module

$$
C \otimes C \stackrel{\omega}{\longrightarrow}\left(C_{2} \vec{*} T\right) *\langle C \otimes C\rangle \stackrel{\delta}{\longrightarrow} D_{4}
$$

where $\omega\left\{b a^{i}\right\} \otimes\left\{b a^{j}\right\}=\left\langle b a^{i} \otimes b a^{j}\right\rangle$ for $i, j=0,1,2$. Moreover we define $u=r_{0} r_{1}=a, v=$ $r_{0}=b$ and $D_{4}^{\prime}=\left\langle u, v: u^{4}=v^{2}=u v u v=1\right\rangle$ the another copy of $D_{4}$. Then we obtain $\delta(u)=a^{2}$ and $\delta(v)=b$ and

$$
C \otimes C \stackrel{\omega}{\longrightarrow} D_{4}^{\prime} *\langle C \otimes C\rangle \stackrel{\delta}{\longrightarrow} D_{4}
$$

is a dihedral reduced quadratic module, where $C$ is the Klein 4-group.

REMARK: Ellis in his paper [14 has stated that crossed squares have a geometric interpretation in terms of relative homotopy groups, but no such interpretation is available for quadratic modules. He also defined a functor $\rho$ from the category of 3-dimensional reduced CW-spaces to the category of crossed squares and showed that $\rho(X)$ is a free crossed square. Furthermore in page 106 of his work [14], obtained a free quadratic module from a free crossed square. Thus Brown and Sivera results for induced crossed squares have immediate topological applications. Of course this description of induced structures for quadratic modules was very useful in situations where a van Kampen theorem gave pushouts arising from topology. The problem with the quadratic module area is that there seems to be no van Kampen type theorem, because there is no direct homotopical functor.

\section{Appendix}

\section{The Proof of Proposition 3.2}

$\left[\bullet^{1}\right]$ We show that the following diagram

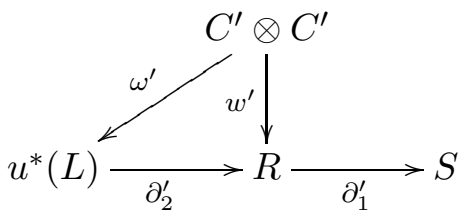

is a quadratic module.

QM1. It is already known that $\partial^{\prime}: R \rightarrow S$ is a nil(2)-module and, since $r \in$ ker $\partial_{1}^{\prime}$, we obtain $\partial_{1}^{\prime} \partial_{2}^{\prime}(r, l)=\partial_{1}^{\prime}(r)=1$. That is,

$$
u^{*}(L) \stackrel{\partial_{2}^{\prime}}{\longrightarrow} R \stackrel{\partial_{1}^{\prime}}{\longrightarrow} S
$$

is a complex of homomorphisms of groups.

QM2. For all $r, r^{\prime} \in R$, we obtain

$$
\partial_{2}^{\prime} \omega^{\prime}\left(\{r\} \otimes\left\{r^{\prime}\right\}\right)=\partial_{2}^{\prime}\left(\left\langle r, r^{\prime}\right\rangle, \omega\left\{u_{1} r\right\} \otimes\left\{u_{1} r^{\prime}\right\}\right)=\left\langle r, r^{\prime}\right\rangle=w^{\prime}\left(\{r\} \otimes\left\{r^{\prime}\right\}\right) .
$$


QM3. For $(r, l) \in u^{*}(L), r^{\prime} \in R$, we obtain

$$
\begin{aligned}
\omega^{\prime}\left(\{ \partial _ { 2 } ^ { \prime } ( r , l ) \} \otimes \left\{\left(r^{\prime}\right)\right.\right. & \left.\left\{r^{\prime}\right\} \otimes\left\{\partial_{2}^{\prime}(r, l)\right\}\right) \\
& =\omega^{\prime}\left(\{r\} \otimes\left\{r^{\prime}\right\}\left\{r^{\prime}\right\} \otimes\{r\}\right) \\
& =\left(\left\langle r, r^{\prime}\right\rangle, \omega\left\{u_{1} r\right\} \otimes\left\{u_{1} r^{\prime}\right\}\right)\left(\left\langle r^{\prime}, r\right\rangle, \omega\left\{u_{1} r^{\prime}\right\} \otimes\left\{u_{1} r\right\}\right) \\
& =\left(\left(r^{-1} r^{\prime-1} r r^{\prime} \partial_{1}^{\prime} r\right)\left(\left(r^{\prime}\right)^{-1} r^{-1}\left(r^{\prime}\right) r^{\partial_{1}^{\prime} r^{\prime}}\right), \omega\left\{\partial_{2} l\right\} \otimes\left\{u_{1} r^{\prime}\right\}\left\{u_{1} r^{\prime}\right\} \otimes\left\{\partial_{2} l\right\}\right) \\
& =\left(\left(r^{-1} r^{\prime-1} r r^{\prime} \partial_{1}^{\prime} r\right)\left(\left(r^{\prime}\right)^{-1} r^{-1}\left(r^{\prime}\right) r_{1}^{\partial_{1}^{\prime} r^{\prime}}\right), l^{-1} l^{\partial_{1} u_{1}\left(r^{\prime}\right)}\right)\left(\text { since } u_{1}(r)=\partial_{2} l\right) \\
& =\left(r^{-1} r^{\partial_{1}^{\prime} r^{\prime}}, l^{-1} l^{\partial_{1} u_{1}\left(r^{\prime}\right)}\right) \quad\left(\text { since } r \in \operatorname{ker} \partial_{1}^{\prime}\right) \\
& =(r, l)^{-1}\left(r^{\partial_{1}^{\prime}\left(r^{\prime}\right)}, l^{u_{0} \partial_{1}^{\prime} r^{\prime}}\right) \quad \\
& =(r, l)^{-1}(r, l)^{\partial_{1}^{\prime} r^{\prime}}
\end{aligned}
$$

QM4. For $(r, l),\left(r^{\prime}, l^{\prime}\right) \in u^{*}(L)$, we get

$$
\begin{aligned}
\omega^{\prime}\left(\left\{\partial_{2}^{\prime}(r, l)\right\} \otimes\left\{\partial_{2}^{\prime}(r, l)\right\}\right) & =\left(\left\langle r, r^{\prime}\right\rangle, \omega\left\{u_{1} r\right\} \otimes\left\{u_{1} r^{\prime}\right\}\right) \\
& =\left(r^{-1} r^{-1} r r^{\prime} \partial_{1}^{\prime} r, \omega\left\{\partial_{2} l\right\} \otimes\left\{\partial_{2} l^{\prime}\right\}\right) \\
& =\left(r^{-1} r^{\prime-1} r r^{\prime},\left[l, l^{\prime}\right]\right) \quad\left(\text { since } r \in \operatorname{ker} \partial_{1}^{\prime}\right) \\
& =\left(\left[r, r^{\prime}\right],\left[l, l^{\prime}\right]\right) \\
& =\left[(r, l),\left(r^{\prime}, l^{\prime}\right)\right] .
\end{aligned}
$$

$\left[\bullet^{2}\right]$ We show that $\left(\varphi, u_{1}, u_{0}\right)$ is a morphism between quadratic modules. Since

$$
u_{1}\left(v_{1} \partial_{2}^{\prime \prime}(z)\right)=u_{1}^{\prime}\left(\partial_{2}^{\prime \prime} z\right)=\partial_{2}(\theta(z))
$$

and $\partial_{1}^{\prime}\left(v_{1} \partial_{2}^{\prime \prime} z\right)=v_{0} \partial_{1}^{\prime \prime} \partial_{2}^{\prime \prime} z=1$, we have $v_{1} \partial_{2}^{\prime \prime}(z) \in \operatorname{ker} \partial_{1}^{\prime}$, and $\left(v_{1} \partial_{2}^{\prime \prime}(z), \theta(z)\right) \in u^{*}(L)$. We obtain

$$
\partial_{2}^{\prime} \psi(z)=\partial_{2}^{\prime}\left(v_{1} \partial_{2}^{\prime \prime}(z), \theta z\right)=v_{1} \partial_{2}^{\prime \prime}(z)
$$

for $z \in Z$ and

$$
\begin{aligned}
\psi\left(\omega^{\prime \prime}\{x\} \otimes\{y\}\right) & =\left(v_{1}\left(\partial_{2}^{\prime \prime} \omega^{\prime \prime}\{x\} \otimes\{y\}\right), \theta\left(\omega^{\prime \prime}\{x\} \otimes\{y\}\right)\right) \\
& =\left(v_{1}(\langle x, y\rangle), \omega\left\{u_{1}^{\prime}(x)\right\} \otimes\left\{u_{1}^{\prime}(y)\right\}\right) \\
& =\left(\left\langle v_{1} x, v_{1} y\right\rangle, \omega\left\{u_{1}\left(v_{1} x\right)\right\} \otimes\left\{u_{1}\left(v_{1} y\right)\right\}\right) \\
& =\omega^{\prime}\left(\left\{v_{1} x\right\} \otimes\left\{v_{1} y\right\}\right)
\end{aligned}
$$

for $\{x\} \otimes\{y\} \in C^{\prime \prime} \otimes C^{\prime \prime}$. 
$\left[\bullet^{3}\right]$ We have for $(n, l) \in F(N \times L)$,

$$
\begin{aligned}
\partial_{1}^{\prime}\left(\partial_{2}^{\prime}\right)(n, l) & =\partial_{1}^{\prime}\left(n^{-1} v_{1} \partial_{2}(l) n\right) \\
& =\partial_{1}^{\prime}\left(n^{-1}\right) \partial_{1}^{\prime} v_{1} \partial_{2}(l) \partial_{1}^{\prime} n \\
& =\partial_{1}^{\prime}\left(n^{-1}\right) v_{0}\left(\partial_{1} \partial_{2} l\right) \partial_{1}^{\prime} n \quad\left(\text { since } \partial_{1}^{\prime} v_{1}=v_{0} \partial_{1}\right) \\
& =\partial_{1}^{\prime}\left(n^{-1}\right) v_{0}(1) \partial_{1}^{\prime} n \quad\left(\text { since } \partial_{1} \partial_{2} l=1\right) \\
& =1
\end{aligned}
$$

and for $\langle\{x\} \otimes\{y\}\rangle \in\langle C \otimes C\rangle$,

$$
\partial_{1}^{\prime}\left(\partial_{2}^{\prime}\langle\{x\} \otimes\{y\}\rangle\right)=\partial_{1}^{\prime}\left(x^{-1} y^{-1} x y^{\partial_{1}^{\prime} x}\right)=1 .
$$

Thus the diagram

$$
F(N \times L) *\langle C \otimes C\rangle \stackrel{\partial_{2}^{\prime}}{\longrightarrow} N \stackrel{\partial_{1}^{\prime}}{\longrightarrow} Q
$$

is a complex of homomorphisms of groups.

$\left[\bullet^{4}\right]$ We show that $\left(\psi, v_{1}, v_{0}\right)$ is a morphism of quadratic modules. We obtain $\partial_{2}^{\prime} \psi(l)=$ $\partial_{2}^{\prime}(1, l)=v_{1} \partial_{2}(l)$ for $l \in L$ and

$$
\psi(\omega\{x\} \otimes\{y\})=(1, \omega\{x\} \otimes\{y\})=\left\langle\left\{v_{1}(x)\right\} \otimes\left\{v_{1}(y)\right\}\right\rangle=\omega^{\prime}\left(\left\{v_{1}(x)\right\} \otimes\left\{v_{1}(y)\right\}\right)
$$

for $x, y \in M$ and

$$
\psi\left(l^{p}\right)=\left(1, l^{p}\right)=\left(v_{0} p, l\right)=(1, l)^{v_{0} p}=\psi(l)^{v_{0} p}
$$

for $p \in P$.

$\left[\bullet^{5}\right]$ We show that $\left(\theta_{*}, i d, i d\right)$ is a morphism of quadratic modules. We obtain for $n \in N$ and $l \in L$

$$
\theta_{*}\left((n, l)^{q}\right)=\theta_{*}\left(n^{q}, l\right)=\left(\theta^{\prime}(l)\right)^{n^{q}}=\left(\theta^{\prime}(l)^{n}\right)^{i d(q)}=\left(\theta_{*}(n, l)\right)^{i d(q)}
$$

and for $x, y \in N$,

$\theta_{*}\left(\langle\{x\} \otimes\{y\}\rangle^{q^{\prime}}\right)=\theta_{*}\left\langle\left\{x^{q^{\prime}}\right\} \otimes\left\{y^{q^{\prime}}\right\}\right\rangle=\omega^{\prime \prime}\left(\left\{x^{q^{\prime}}\right\} \otimes\left\{y^{q^{\prime}}\right\}\right)=\omega^{\prime \prime}(\{x\} \otimes\{y\})^{q^{\prime}}=\left(\theta_{*}\langle\{x\} \otimes\{y\}\rangle\right)^{i d\left(q^{\prime}\right)}$

Further, we obtain

$$
\begin{aligned}
\partial_{2}^{\prime \prime}\left(\theta_{*}(n, l)\right) & =\partial_{2}^{\prime \prime}\left(\theta^{\prime}(l)^{n}\right) \\
& =n^{-1}\left(\partial_{2}^{\prime \prime}\left(\theta^{\prime}(l)\right)\right) n \\
& =n^{-1}\left(v_{1} \partial_{2}(l)\right) n \\
& =i d\left(\partial_{2}^{\prime}(n, l)\right)
\end{aligned}
$$

and

$$
\begin{aligned}
\partial_{2}^{\prime \prime}\left(\theta_{*}\langle\{x\} \otimes\{y\}\rangle\right) & =\partial_{2}^{\prime \prime}\left(\omega^{\prime \prime}\{x\} \otimes\{y\}\right) \\
& =w(\{x\} \otimes\{y\}) \\
& =x^{-1} y^{-1} x y_{1}^{\partial_{1}^{\prime} x} \\
& =\partial_{2}^{\prime}\langle\{x\} \otimes\{y\}\rangle
\end{aligned}
$$


for $(n, l) \in F(N \times L)$ and $\langle\{x\} \otimes\{y\}\rangle \in\langle C \otimes C\rangle$. Therefore, $\left(\theta_{*}, i d, i d\right)$ is the unique morphism in $\operatorname{Quad}_{(N \rightarrow Q)}$.

\section{References}

[1] U. E. Arslan, Z. Arvasi and G. Onarli, (Co)-Induced two-crossed modules, arXiv:1107.4291v3 [math.AT] 11 Feb 2013, available from http://arxiv.org/pdf/1107.4291v3.pdf.

[2] Z. Arvasi and E. Ulualan, Freeness conditions for quadratic modules and quadratic chain complexes, Georgian Math. J., 18, 615-637, (2011).

[3] Z. Arvasi and E. Ulualan, On algebraic models for homotopy 3-types, Journal of Homotopy and Related Structures 1, 1, 1-27, (2006).

[4] H.J. Baues, Combinatorial homotopy and 4-dimensional complexes, Walter de Gruyter, 15, 380 pages, (1991).

[5] R. Brown and P. J. Higgins, On the connection between the second relative homotopy groups of some related spaces, Proc. London Math. Soc., (3), 36, (2), 193-212, (1978).

[6] R. Brown, P. J. Higgins and R. Sivera, Nonabelian algebraic topology: filtered spaces, crossed complexes, cubical higher homotopy groupoids, available from http://www.bangor.ac.uk/ mas010/pdffiles/rbrsbookb-e231109.pdf.

[7] R. Brown and J.-L. Loday, Homotopical excision, and Hurewicz theorems for n-cubes of spaces, Proc. London Math. Soc. (3), 54, (1), (1987).

[8] R. Brown and R. Sivera, Algebraic colimit calculations in homotopy theory using fibred and cofibred categories, Theory and Applications of Categories, 22, 222-251, (2009).

[9] R. Brown and C. D. Wensley, On finite induced crossed modules, and the homotopy 2-type of mapping cones, Theory and Applications of Categories, (3) 1 (1995), 54-71.

[10] R. Brown and C. D. Wensley, Computation and homotopical applications of induced crossed modules, Journal of Symbolic Computation 35, 2003, 59-72.

[11] J.M. CASAS and M. LADra, Colimits in the crossed modules category in Lie algebras, Georgian Mathematical Journal, 7, 3, 461-474, (2000).

[12] D. Conduché, Modules croisés généralisés de longueur 2, Journal of Pure and Applied Algebra, 34, 155-178, (1984).

[13] A. Grothendieck, Catégories cofibr'ees additives et complexe cotangent relatif, Lecture Notes in Mathematics, 79. Springer-Verlag, Berlin (1968). 
[14] G.J. ElLIS, Crossed squares and combinatorial homotopy, Math.Z., 214, 93-110, (1993).

[15] D. Guin-Walery and J.L. Loday, Obsruction á l'excision en K-theories algébrique, In: Friedlander, E.M.,Stein, M.R.(eds.) Evanston conf. on algebraic K-Theory 1980. (Lect. Notes Math., vol.854, 179-216) Berlin Heidelberg New York: Springer (1981).

[16] J.L. Loday, Spaces with finitely many non-trivial homotopy groups , J. Pure and Applied Algebra, 24, 179-202, (1982).

[17] F. Muro, Suspensions of crossed and quadratic complexes, Co-H-stuctures and applications, Trans. Amer. Math. Soc., 357, 3623-3653, (2005).

[18] T. Porter, Some categorical results in the theory of crossed modules in commutative algebras, Journal of Algebra, 109, 415-429, (1987).

[19] T. Porter, Homotopy Quantum Field Theories meets the Crossed Menagerie: an introduction to HQFTs and their relationship with things simplicial and with lots of crossed gadgetry. Notes prepared for the Workshop and School on Higher Gauge Theory, TQFT and Quantum Gravity Lisbon, February, 2011, available from http://ncatlab.org/timporter/files/HQFT-XMenagerie.pdf

[20] T. Streicher, Fibred categories a la Bénabou, available from http://www.mathematik.tu-darmstadt.de/ streicher/FIBR/FibLec.pdf, 1-94, April 1999- February 2012.

[21] J.H.C. Whitehead, Combinatorial homotopy II, Bull. Amer. Math. Soc., 55, 453-496, (1949).

\author{
Hasan ATIKK \\ İstanbul Medeniyet University \\ Science Faculty \\ Mathematics Department \\ İstanbul, TURKEY \\ hasanatik@yahoo.com
}

\author{
Erdal ULUALAN \\ Dumlupinar University \\ Science and Art Faculty \\ Mathematics Department \\ Kütahya, TURKEY \\ eulualan@gmail.com, erdal.ulualan@dpu.edu.tr
}

